\title{
Noise-driven dynamics and phase transitions in fermionic systems
}

\author{
Birger Horstmann, ${ }^{1,2}$ J. Ignacio Cirac, ${ }^{1}$ and Géza Giedke ${ }^{1,3}$ \\ ${ }^{1}$ Max-Planck-Institut für Quantenoptik, Hans-Kopfermann-Straße 1, 85748 Garching, Germany \\ ${ }^{2}$ Deutsches Zentrum für Luft- und Raumfahrt, Institut für Technische Thermodynamik, Pfaffenwaldring 38-40, 70569 Stuttgart, Germany \\ ${ }^{3}$ Zentrum Mathematik, Technische Universität München, L.-Boltzmannstrasse 3, 85748 Garching, Germany
}

(Received 3 August 2012; published 10 January 2013)

\begin{abstract}
We study abrupt changes in the dynamics and/or steady state of fermionic noise-driven systems produced by small changes in the system parameters. Specifically, we consider fermionic systems whose dynamics is described by master equations that are quadratic (and, under certain conditions, quartic) in creation and annihilation operators. We analyze phase transitions in the steady state as well as "dynamical transitions." The latter are characterized by abrupt changes in the rate at which the system asymptotically approaches the steady state. We illustrate our general findings with relevant examples of fermionic (and, equivalently, spin) systems and show that they can be realized in ion chains.
\end{abstract}

DOI: 10.1103/PhysRevA.87.012108

PACS number(s): 03.65.Yz, 05.30.Rt

\section{INTRODUCTION}

Motivated by the impressive experimental control over many-body quantum states and dynamics [1], open manybody quantum systems have received increasing experimental and theoretical attention in recent years. On one hand, the decoherence introduced by coupling to an environment or by quantum noise is a major challenge to quantum information processing [2], on the other hand, it can play a constructive role for quantum computing [3,4], state preparation [5-8], entanglement generation [9,10], quantum memories [11], or quantum simulation [12-16].

These exciting possibilities drive the interest in understanding the steady-state phase diagram of open systems in detail [17]. Of particular interest are points of transitions between different phases of the system. For closed systems at zero temperature, the phase diagram and quantum phase transition can be understood by studying the low-lying energy eigenstates of the system's Hamiltonian [18]. In particular, the nonanalyticity of certain expectation values as a function of an external parameter, that characterizes the quantum phase transition, can only occur if the gap of the Hamiltonian closes, i.e., the energy difference between ground state and first excited state vanishes. Quantum phase transitions are, thus, determined by the low-energy spectrum of the Hamiltonian governing the dynamics of wave functions,

$$
\partial_{t}|\Phi\rangle=-\frac{i}{\hbar} \mathbf{H}|\Phi\rangle
$$

In this paper, we study abrupt changes in the physical properties of a many-body quantum system whose dynamics is described by a master equation,

$$
\partial_{t} \rho=\mathcal{S} \rho .
$$

This equation describes the dynamics of an open system coupled to a Markovian reservoir where $\rho$ is the system's density operator. The superoperator $\mathcal{S}$ contains two parts: One is related to the system Hamiltonian (possibly renormalized due to the interaction with the environment), and the other is related to the quantum noise induced by the environment. Under the appropriate conditions, the system evolves to a steady state $\rho_{\text {ss }}$, which corresponds to a (right) eigenstate of $\mathcal{S}$ with eigenvalue 0 . Note that this eigenvalue may be degenerate, or there may be other eigenvalues with zero real parts. In case this does not happen, the steady state is unique. Then, the other eigenvalues $\lambda$ of $\mathcal{S}$ have a negative real part, and the smallest absolute value of them $\Delta$ determines the asymptotic decay rate (ADR), that is, the rate at which the steady state is reached. A phase transition in the steady state, where its properties abruptly change when one slightly changes a parameter in the master equation, is accompanied by the vanishing of $\Delta$. This situation has recently been studied by many authors (see, for example, Refs. $[3,5,17,19,20])$ and is typically referred to as a "dissipative quantum phase transition" or "noise-driven quantum phase transition." There is a natural analogy between noise-driven and (closed-system) quantum phase transitions: A unique ground state of the Hamiltonian is analogous to a unique steady state. The appearance of a phase transition is signaled by the vanishing of the gap or $\Delta$, respectively.

Apart from its role in reflecting the appearance of a phase transition, the quantity $\Delta$ can play an additional role. It also represents a physical property of the system, namely, the rate at which the steady state is approached asymptotically or the system's response to perturbations in the steady state. This quantity may change abruptly itself. In that case, we can talk about a dynamical transition since a small change in the system parameters may lead to an abrupt change in the dynamics of the system. Actually, such a transition may, in principle, occur even if $\Delta$ remains finite, and thus, it is a different property than the transitions generally studied in this context.

In this paper, we investigate both kinds of transitions for simple fermionic systems. We concentrate on systems that are described by master equations and for which the Hamiltonian is, at most, quadratic in fermionic creation and annihilation operators. Specifically, we consider two kinds of noise terms: (i) general quadratic in the fermionic operators and (ii) quartic, but with some conditions (namely, that they correspond to Hermitian Lindblad operators). In the first case, the dynamics can be exactly solved [21-24], which has been exploited in several recent papers to study the interplay of noise and critical Hamiltonians in one-dimensional (1D) fermionic systems $[19,23,25]$. In the second case, even though the full dynamics cannot be obtained, we show that it is, nevertheless, possible to exactly determine the dynamics of certain expectation values 
from which dynamical and steady-state properties can be obtained. In this last case, we present analytical examples where dynamical transitions occur [26]. This situation has also been studied in Refs. [27-29] with particular regard to transport through a dephasing spin chain where exact solutions of the associated master equation could be obtained.

The formalism we develop is relatively general, and we illustrate it with explicit examples. In particular, we consider Hamiltonians which are intimately connected to physical situations that can be obtained in the laboratory, namely, anisotropic $X Y$ spin chains in transverse magnetic fields and that are mapped to a fermionic Hamiltonian by a JordanWigner transformation. This family of Hamiltonians displays the prototype of a continuous phase transition [18]. The noise terms we consider can also be understood as particular physical processes occurring in the spin chain through its interaction with an environment [30]. Note that our framework also applies to the systems studied in Refs. [21,22,27,28] and, for the quadratic noise terms, is related to Refs. [19,23] where generic noise-driven phase transitions are analyzed.

This paper is structured as follows. In Sec. II, we introduce the Lindblad master equation, which allows describing decoherence due to the weak interaction with a Markovian bath and presents the covariance matrix (CM) formalism, which allows the exact treatment of quadratic fermionic systems. In Sec. III, we extend this formalism to decoherent systems with linear and Hermitian quadratic Lindblad operators. Then, we come to the calculation of the steady states and the ADRs for relevant interesting examples in this framework in Secs. IV-VI. Here, we explicitly demonstrate the presence of noise-driven phase transitions. In Sec. VII, we propose a possible implementation with cold ions before concluding in Sec. VIII.

\section{NOTATION AND METHODS}

In this section, we introduce our tools and notation, namely, the Lindblad master equation and the fermionic CM formalism, which is ideally suited for describing quasifree fermionic systems (see Sec. II C).

\section{A. Lindblad master equation}

We consider systems whose interaction with an environment leads to a time evolution governed by a Lindblad master equation [31],

$$
\partial_{t} \rho=\mathcal{S} \rho=-\frac{i}{\hbar}[\mathbf{H}, \rho]+\sum_{\alpha}\left(\mathbf{L}^{\alpha} \rho \mathbf{L}^{\alpha \dagger}-\frac{1}{2}\left\{\mathbf{L}^{\alpha \dagger} \mathbf{L}^{\alpha}, \rho\right\}\right),
$$

where $\rho$ is the density matrix of the system, $\mathbf{H}$ is its Hamiltonian, and the Lindblad operators $\mathbf{L}^{\alpha}$ determine the interaction between the system and the bath. This dynamical equation for an open system can be derived from two different points of view [32]: First, it can be derived from the full dynamics of system and bath. Here, three major approximations have to be used: The states of system and environment are initially uncorrelated, the coupling between system and bath is weak (Born approximation), and the environment equilibrates fast (Markov approximation). Second, any time evolution given by a quantum dynamical semigroup (i.e., a family of completely positive trace-preserving maps $\epsilon_{t}$, which is strongly continuous and satisfies $\epsilon_{t} \epsilon_{s}=\epsilon_{t+s}$ ) is generated by an equation of the form Eq. (3).

We characterize the decoherence dynamics with the steady state and the asymptotic decay rate. A steady-state density matrix $\rho_{0}$ of the master equation (3) fulfills

$$
\partial_{t} \rho_{0}=\mathcal{S} \rho_{0}=0
$$

and is the (generically unique) eigenvector with eigenvalue 0 of the Liouvillian superoperator $\mathcal{S}$. The approach to the steady state is then governed by the nonzero eigenvalues (and eigenvectors) of $\mathcal{S}$, all of which have nonpositive real parts for Liouvillians of Lindblad form. Of particular interest is the eigenvalue with the largest real part since it governs the long-term dynamics. We refer to the absolute value of this largest real part as the ADR and denote it by $\Delta$,

$$
\Delta(\mathcal{S})=\max \left\{|\operatorname{Re} \lambda| \neq 0: \exists \rho_{\lambda}: \mathcal{S}\left(\rho_{\lambda}\right)=\lambda \rho_{\lambda}\right\}
$$

\section{B. Quasifree fermions and spins}

We consider systems with $N$ fermionic modes described by creation and annihilation operators $a_{j}^{\dagger}$ and $a_{j}$. These operators obey the canonical anticommutation relations,

$$
\left\{a_{j}, a_{k}\right\}=0, \quad\left\{a_{j}^{\dagger}, a_{k}\right\}=\delta_{j k} .
$$

Equivalently, we can use Hermitian fermionic Majorana operators,

$$
c_{j, 0}=a_{j}^{\dagger}+a_{j}, \quad c_{j, 1}=(-i)\left(a_{j}^{\dagger}-a_{j}\right),
$$

which, as generators of the Clifford algebra, satisfy the anticommutation relations,

$$
\left\{c_{j, u}, c_{k, v}\right\}=2 \delta_{j k} \delta_{u v} .
$$

We consider fermionic Hamiltonians that are quadratic in the Majorana operators. They describe quasifree fermions and are known to be exactly solvable. We parametrize them with the real antisymmetric matrix $H$,

$$
\mathbf{H}=\frac{i}{4} \hbar \sum_{j k u v} H_{j k, u v} c_{j, u} c_{k, v} .
$$

The $2 \times 2$ matrix $H_{j k} \equiv\left(H_{j k, u v}\right)_{u v}$ describes the coupling between modes $j$ and $k$.

All eigenstates and thermal states of such a quadratic fermionic Hamiltonian are Gaussian, i.e., they have a density operator, which is the exponential of a quadratic form in the Majorana operators. Gaussian states remain Gaussian under the evolution with quadratic Hamiltonians.

In the following, we will mostly be concerned with translationally invariant systems and nearest-neighbor interactions. In terms of the matrix $H$, the former means that $H_{j k}$ depends only on the difference $j-k$, and for short, we write

$$
H_{j k} \equiv H_{j-k},
$$

whereas, the latter implies that $H_{s}=0$ for $s>1$. We work with periodic boundary conditions, so $j-k$ is understood $\bmod N$.

An important reason to study one-dimensional fermionic systems with quadratic Hamiltonians is their intimate relation 
to certain types of spin chains: The Jordan-Wigner transformation [33] maps fermionic operators onto Pauli spin operators via

$$
c_{j, 0} \leftrightarrow \prod_{k=1}^{j-1} \sigma_{z}^{k} \sigma_{x}^{j}, \quad c_{j, 1} \leftrightarrow \prod_{k=1}^{j-1} \sigma_{z}^{k} \sigma_{y}^{j} .
$$

Under this transformation, some spin chains are mapped to spinless quasifree fermionic systems, which can be solved exactly. A prominent example is the anisotropic $X Y$ chain in a transverse magnetic field [18] with the Hamiltonian,

$$
\begin{aligned}
\mathbf{H}= & -J \sum_{j=1}^{N}\left[(1+\gamma) \sigma_{x}^{j} \sigma_{x}^{j+1}+(1-\gamma) \sigma_{y}^{j} \sigma_{y}^{j+1}\right] \\
& +B \sum_{j=1}^{N} \sigma_{z}^{j}
\end{aligned}
$$

where $B$ is the magnetic field, $J$ is the ferromagnetic coupling, and $\gamma$ is the anisotropy parameter. Closed systems governed by this Hamiltonian show a quantum phase transition at $B=2 J$ in the thermodynamic limit, and the behavior in the presence of noise is studied in Sec. VIB.

We are interested in noise-driven open fermionic systems with dynamics described by a Lindblad master equation, characterized by a set of Lindblad operators $L^{\alpha}$. We consider two classes of Lindblad operators: first, those given by arbitrary linear combinations of the Majorana operators (linear Lindblad operators),

$$
\mathbf{L}^{\alpha}=\sum_{j u} L_{j, u}^{\alpha} c_{j, u}, \quad L_{j, u}^{\alpha} \in \mathbb{C},
$$

and second, those represented by quadratic expressions in the Majorana operators, which are, in addition, Hermitian (Hermitian quadratic Lindblad operators),

$$
\mathbf{L}^{\alpha}=\frac{i}{4} \sum_{j k u v} L_{j k, u v}^{\alpha} c_{j, u} c_{k, v},
$$

with the real and antisymmetric matrix $L^{\alpha}$.

\section{Covariance matrix formalism}

Now, we present a framework in which the noise-driven dynamics of the Lindblad master equation (3) can be solved exactly.

For every state of a fermionic system, its real and antisymmetric CM is defined by

$$
\Gamma_{j k, u v}=\operatorname{tr}\left(\rho \frac{i}{2}\left[c_{j, u}, c_{k, v}\right]\right) .
$$

The magnitudes of the imaginary eigenvalues of $\Gamma$ are smaller than or equal to unity $\left(\Gamma^{2} \leqslant-\mathbb{1}\right)$.

For Gaussian states, the correlation functions of all orders are related to the CM through Wick's theorem [34]. In particular, pure Gaussian states $\rho=|\Psi\rangle\langle\Psi|$ satisfy $\Gamma^{2}=-\mathbb{1}$. In our notation, $\Gamma_{j k}$ denotes a $2 \times 2$ matrix that describes the covariances between sites $j$ and $k$.

\section{LINDBLAD MASTER EQUATION IN THE COVARIANCE MATRIX FORMALISM}

The CM formalism is especially useful if the operative dynamics leads to closed equations for the CM, which is the case for the two kinds of Lindblad operators Eqs. (13) and (14) that we study in the following.

\section{A. Linear Lindblad operators}

We consider a system with a quadratic Hamiltonian given by the antisymmetric matrix $H$ [cf. Eq. (9)] and linear Lindblad operators as defined in Eq. (13). Using the anticommutation relations (8), we determine the dynamical equation for the $\mathrm{CM}$ $\Gamma$ from Eq. (3) and obtain

$$
\begin{aligned}
\partial_{t} \Gamma= & {[H, \Gamma]-\sum_{\alpha}\left\{\left|L^{\alpha}\right\rangle\left\langle L^{\alpha}|+| L^{\alpha *}\right\rangle\left\langle L^{\alpha *}\right|, \Gamma\right\} } \\
& -2 i\left(\left|L^{\alpha}\right\rangle\left\langle L^{\alpha}|-| L^{\alpha *}\right\rangle\left\langle L^{\alpha *}\right|\right),
\end{aligned}
$$

where $\left|L^{\alpha}\right\rangle$ denotes the vector formed by the coefficients $L_{j, u}^{\alpha}$ in Eq. (13) and $\left|L^{\alpha *}\right\rangle$ denotes its complex conjugate. In terms of $|\Gamma\rangle$, the vector of components of $\Gamma$, this equation becomes

$$
\partial_{t}|\Gamma\rangle=\mathcal{S}|\Gamma\rangle-|\mathcal{V}\rangle=(\mathcal{H}-\mathcal{M})|\Gamma\rangle-|\mathcal{V}\rangle,
$$

with the superoperators,

$$
\begin{gathered}
\mathcal{H}=\left(H \otimes \mathbb{1}-\mathbb{1} \otimes H^{T}\right), \\
\mathcal{M}=\sum_{\alpha}\left[\left|L^{\alpha}\right\rangle\left\langle L^{\alpha}\right| \otimes \mathbb{1}+\mathbb{1} \otimes\left(\left|L^{\alpha}\right\rangle\left\langle L^{\alpha}\right|\right)^{T}+\text { c.c. }\right], \\
|\mathcal{V}\rangle=2 i \sum_{\alpha}\left(\left|L^{\alpha}\right\rangle \otimes\left|L^{\alpha}\right\rangle-\text { c.c. }\right) .
\end{gathered}
$$

Note that $\mathcal{H}$ is anti-Hermitian and $\mathcal{M}$ is Hermitian and positive semidefinite. The steady-state CM [see Eq. (4)] satisfies

$$
(\mathcal{H}-\mathcal{M})\left|\Gamma_{0}\right\rangle=|\mathcal{V}\rangle \text {. }
$$

Deviations $|\delta \Gamma\rangle=|\Gamma\rangle-\left|\Gamma_{0}\right\rangle$ then obey

$$
\partial_{t}|\delta \Gamma\rangle=(\mathcal{H}-\mathcal{M})|\delta \Gamma\rangle,
$$

and the approach to the steady state is governed by the the right eigenvalues of the superoperator $\mathcal{S}=\mathcal{H}-\mathcal{M}$, satisfying

$$
\mathcal{S}\left|\Gamma_{i}\right\rangle=\lambda_{i}\left|\Gamma_{i}\right\rangle
$$

The eigenvalues whose real parts are closest to zero, thus, determine the asymptotics of the decoherence process. In the following, we refer to

$\Delta=\max \left\{\left|\operatorname{Re} \lambda_{i}\right| \neq 0: \exists \Gamma_{i} \quad\right.$ such that $\left.\left(\mathcal{S}-\lambda_{i}\right)\left|\Gamma_{i}\right\rangle=0\right\}$,

i.e., the asymptotic decay rate on the level of CMs simply as ADR.

\section{B. Quadratic and Hermitian Lindblad operators}

The second class of master equations, leading to closed equations for the CM, is of the form Eq. (3) with Lindblad operators that are quadratic and Hermitian as in Eq. (14). Lindblad equations with Hermitian Lindblad operators describe the dynamics of systems in contact with a classical bath. Let us choose a fluctuating external field as the source of decoherence (see Sec. VII). If, additionally, the Lindblad operators are 
quadratic, the fluctuating Hamiltonian is quadratic. Thus, in this case, Gaussian states evolve into mixtures of Gaussian states under such evolutions, and we can expect a closed equation for the $\mathrm{CM}$.

Before discussing the master equation in the CM formalism, let us first determine, in general, the steady-state density matrices [see Eq. (4)] of a master equation with only Hermitian Lindblad operators. In that case, we can rewrite the master equation in terms of $|\rho\rangle$, the vector of components of $\rho$ as

$$
\partial_{t}|\rho\rangle=\mathcal{S}|\rho\rangle=\left(\mathcal{H}-\frac{1}{2} \sum_{\alpha}\left(\mathcal{L}^{\alpha}\right)^{2}\right)|\rho\rangle,
$$

with the superoperators,

$$
\begin{gathered}
\mathcal{H}=-i\left(\mathbf{H} \otimes \mathbf{1}-\mathbf{1} \otimes \mathbf{H}^{T}\right), \\
\mathcal{L}^{\alpha}=\mathbf{L}^{\alpha} \otimes \mathbf{1}-\mathbf{1} \otimes \mathbf{L}^{\alpha T} .
\end{gathered}
$$

We observe that the superoperator $\mathcal{H}$ is anti-Hermitian and that the superoperators $\mathcal{L}^{\alpha}$ are Hermitian so that the $\left(\mathcal{L}^{\alpha}\right)^{2}$ are Hermitian and non-negative.

We consider all complex-valued vectors $|\rho\rangle$ instead of just the ones corresponding to positive density matrices with trace 1. Therefore, we have to check, after the calculation, if our results correspond to physically meaningful states. The steady states satisfy

$$
\left\langle\rho_{0}\left|\left(\mathcal{H}-\frac{1}{2} \sum_{\alpha}\left(\mathcal{L}^{\alpha}\right)^{2}\right)\right| \rho_{0}\right\rangle=0
$$

As stated above, $\mathcal{H}$ is anti-Hermitian, and all $\left(\mathcal{L}^{\alpha}\right)^{2}$ are Hermitian. Applying these properties, we can conclude from Eq. (28) that

$$
\left\langle\rho_{0}\left|\sum_{\alpha}\left(\mathcal{L}^{\alpha}\right)^{2}\right| \rho_{0}\right\rangle=\left\langle\rho_{0}|\mathcal{H}| \rho_{0}\right\rangle=0
$$

holds. It follows from the non-negativity of $\left(\mathcal{L}^{\alpha}\right)^{2}$ that

$$
\left(\mathcal{L}^{\alpha}\right)^{2}\left|\rho_{0}\right\rangle=0 \quad \forall \alpha .
$$

Because the $\mathcal{L}^{\alpha}$ can be diagonalized, this implies $\mathcal{L}^{\alpha}\left|\rho_{0}\right\rangle=0$. It follows that $\mathcal{H}\left|\rho_{0}\right\rangle$ vanishes identically. In terms of matrices $\rho_{0}$, we can summarize these conditions for steady states,

$$
\left[\mathbf{H}, \rho_{0}\right]=\left[\mathbf{L}^{\alpha}, \rho_{0}\right]=0 \quad \forall \alpha .
$$

It can be verified, with Eq. (3), that this condition for steady states is not only necessary, but also is sufficient. To summarize, steady states for Hermitian Lindblad operators correspond to density matrices commuting with the Hamiltonian and all Lindblad operators. Therefore, they are the identity up to symmetries shared by the Hamiltonian and the Lindblad operators.

Let us now return to exactly solvable systems in the CM formalism. For quadratic and Hermitian Lindblad operators and quadratic Hamiltonians, the master equation (3) becomes

$$
\partial_{t} \Gamma=[H, \Gamma]+\frac{1}{2} \sum_{\alpha}\left[L^{\alpha},\left[L^{\alpha}, \Gamma\right]\right] .
$$

We can again reformulate this equation for the vector of components $|\Gamma\rangle$,

$$
\partial_{t}|\Gamma\rangle=S|\Gamma\rangle=\left(\mathcal{H}-\frac{1}{2} \sum_{\alpha}\left(\mathcal{L}^{\alpha}\right)^{2}\right)|\Gamma\rangle,
$$

with $\mathcal{H}$ as in Eq. (18) and $\mathcal{L}^{\alpha}=L^{\alpha} \otimes \mathbb{1}-\mathbb{1} \otimes L^{\alpha}$.

Since we found that steady states are trivial for Hermitian Lindblad operators, we concentrate on the asymptotics of the decoherence process. It is studied through the eigenvalues $\lambda_{i}$ of the superoperator $\mathcal{S}$ and, in particular, its ADR as defined in Eq. (24).

\section{Translationally invariant Hamiltonians}

Naturally, translationally invariant systems are best treated in a Fourier-transformed picture. Any real antisymmetric matrix can be transformed into a real and antisymmetric block-diagonal matrix by an orthogonal transformation $O$. For the Hamiltonian matrix $H$, this means

$$
H_{m n, u v}^{\prime}=\left(O H O^{T}\right)_{m n, u v}, \quad H_{m n}^{\prime}=\delta_{m n}\left(\begin{array}{cc}
0 & \epsilon_{m} \\
-\epsilon_{m} & 0
\end{array}\right) \text {, }
$$

where the real number $\epsilon_{m}$ is the energies of the elementary excitations. We, however, transform the Hamiltonian matrix with the unitary Fourier transform,

$$
\widetilde{H}_{m n, u v}=\left(U H U^{\dagger}\right)_{m n, u v}, \quad U_{m n, u v}=\frac{1}{\sqrt{N}} e^{(2 \pi i / N) m n} \delta_{u v} .
$$

The resulting matrix $\widetilde{H}$ is anti-Hermitian but not real. For translationally invariant systems, for which the $2 \times 2$ matrices $H_{j k}$ in Eq. (9) depend only on $j-k$, the matrix $\widetilde{H}$ is block diagonal with

$$
\widetilde{H}_{m n}=\delta_{m n} \sum_{s=0}^{N-1} H_{s} e^{-(2 \pi i / N) s m} .
$$

The block diagonal is parametrized according to

$$
\widetilde{H}_{n n}=\left(\begin{array}{cc}
i k_{n} & h_{n} \\
-h_{n}^{*} & i l_{n}
\end{array}\right), \quad k_{n}, l_{n} \in \mathbb{R}, \quad h_{n} \in \mathbb{C} .
$$

For later use, we observe the properties,

$$
h_{-n}=h_{n}^{*}, \quad k_{-n}=-k_{n}, \quad l_{-n}=-l_{n},
$$

which follow directly from Eq. (36) for real $H_{s}$.

For a system that is also invariant under reflections (in real space), $H_{s}=-H_{s}^{T}$ holds (in addition to $H_{-s}=-H_{s}^{T}$ implied by antisymmetry). In that case, we have $\tilde{H}_{n n}=-\tilde{H}_{n n}$, and therefore,

$$
k_{n}=l_{n}=0 .
$$

The spectrum of the Hamiltonian matrix determines the elementary excitation energies,

$$
\epsilon_{n}=\left|\frac{k_{n}+l_{n}}{2} \pm \sqrt{\left(\frac{k_{n}-l_{n}}{2}\right)^{2}+\left|h_{n}\right|^{2}}\right| .
$$

It will be necessary to transform the $\mathrm{CM} \Gamma$ accordingly, defining

$$
\widetilde{\Gamma}=U \Gamma U^{\dagger}
$$


By minimizing the energy expectation value,

$$
\langle E\rangle=\operatorname{Tr}\left(H^{T} \Gamma\right)=\operatorname{Tr}\left(\widetilde{H}^{\dagger} \widetilde{\Gamma}\right),
$$

we find the CM for the ground state. In the case of $k_{n} l_{n}<\left|h_{n}\right|^{2}$, it is

$$
\widetilde{\Gamma}_{m n}^{0}=\delta_{m n}\left\{\left[\left(k_{n}-l_{n}\right) / 2^{2}\right]+\left|h_{n}\right|^{2}\right\}^{-1 / 2}\left(\begin{array}{cc}
i \frac{k_{n}-l_{n}}{2} & -h_{n} \\
h_{n}^{*} & -i \frac{k_{n}-l_{n}}{2}
\end{array}\right),
$$

and, otherwise,

$$
\widetilde{\Gamma}_{m n}^{0}=-i \delta_{m n} \operatorname{sgn}\left(k_{n}+l_{n}\right) \mathbb{1}_{2} .
$$

For translationally invariant and reflection symmetric systems, $k_{n} l_{n}=0$ holds, thus, $k_{n} l_{n}<\left|h_{n}\right|^{2}$ is fulfilled in such systems. Since the $X Y$ chain Eq. (12) is reflection symmetric, we can concentrate on the case of Eq. (43). Specifically, we obtain, for the Hamiltonian Eq. (12), that

$$
h_{n}=-2 B+2 J\left[(1+\gamma) e^{(2 \pi i / N) n}+(1-\gamma) e^{-(2 \pi i / N) n}\right],
$$

$$
k_{n}=l_{n}=0,
$$

which contains a continuous quantum phase transition at $B=$ $2 J$ where the gap closes and an elementary excitation energy $\epsilon_{n}=\left|h_{n}\right|=0$ exists. This Hamiltonian will be discussed further in Sec. VI.

\section{LINEAR LINDBLAD OPERATORS}

Now, we apply the formalism introduced in the previous sections to some simple cases of physical interest. Here, we choose the simplest examples, i.e., linear Lindblad operators (see Sec. III A). We study two settings. In Sec. IV A, we look at systems without any unitary evolution, observing dynamic transitions when tuning the strength of competing decoherence processes. Here, we enrich our presentation with an example for noise-driven state engineering. In Sec. IV B, we consider systems governed by a Hamiltonian, which describes a quantum phase transition itself and are subject to noise and show that the noise-driven system undergoes a transition for the same values of the system parameters.

\section{A. Purely noise-driven systems}

The simplest example of two competing decoherence processes generated by linear Lindblad operators is

$$
\mathbf{L}_{-}^{\alpha}=g \mu a_{\alpha}, \quad \mathbf{L}_{+}^{\alpha}=g v a_{\alpha}^{\dagger},
$$

acting on site $\alpha \in\{1, \ldots, N\}$. It describes the competition between particle-loss and particle-gain processes. We observe that the master equation (16), without the Hamiltonian $(H=$ 0 ), is diagonal in real space,

$$
\partial_{t} \Gamma=-g^{2}\left(\mu^{2}+v^{2}\right) \Gamma-g^{2}\left(\mu^{2}-v^{2}\right) \bigoplus_{\alpha=1}^{N}\left(i \sigma_{y}\right) .
$$

In this simple case, the master equation is already diagonal, and we read off the single decoherence rate $\Delta=g^{2}\left(\mu^{2}+v^{2}\right)$. Solving the master equation for $\partial_{t} \Gamma_{0}=0$ gives the unique steady-state CM,

$$
\Gamma_{0}=-\frac{\mu^{2}-v^{2}}{\mu^{2}+v^{2}} \bigoplus_{\alpha=1}^{N}\left(\begin{array}{cc}
0 & 1 \\
-1 & 0
\end{array}\right),
$$

which is block diagonal. This state is characterized by the particle number $\left\langle a_{\alpha}^{\dagger} a_{\alpha}\right\rangle=v^{2} /\left(\mu^{2}+v^{2}\right)$ at all sites. For pure particle-loss processes $(v=0)$, all sites are unoccupied $\left\langle a_{\alpha}^{\dagger} a_{\alpha}\right\rangle=0$ in the steady state, whereas, for pure particle-gain processes $(\mu=0)$, all sites are occupied $\left\langle a_{\alpha}^{\dagger} a_{\alpha}\right\rangle=1$. At $\mu=v$, the steady state is the unpolarized completely mixed state. Not surprisingly, the system does not display any phase transition.

More interesting may be the case in which noise can also induce correlations. A simple example of this kind is provided by the Lindblad operators,

$$
\mathbf{L}^{\alpha}=g\left(\mu a_{\alpha}+v a_{\alpha+1}^{\dagger}\right),
$$

acting on nearest neighbors. This set of Lindblad operators generates a master equation, which is diagonal after the Fourier transform (35),

$$
\begin{aligned}
\partial_{t} \widetilde{\Gamma}= & -g^{2}\left(\mu^{2}+v^{2}\right) \widetilde{\Gamma}-g^{2} \mu \nu\left\{\bigoplus_{n=1}^{N} \cos (2 \pi n / N) \sigma_{z}, \widetilde{\Gamma}\right\} \\
& -g^{2}\left(\mu^{2}-v^{2}\right) \bigoplus_{n=1}^{N} i \sigma_{y}-2 g^{2} \mu \nu \bigoplus_{n=1}^{N} i \sin (2 \pi n / N) \sigma_{x} .
\end{aligned}
$$

In this case, a spectrum of decoherence rates $g^{2}\left\{\mu^{2}+v^{2} \pm\right.$ $\left.2 \mu \nu\left[\cos \frac{2 \pi n}{N}+\cos \frac{2 \pi m}{N}\right], \mu^{2}+v^{2} \pm 2 \mu \nu\left[\cos \frac{2 \pi n}{N}-\cos \frac{2 \pi m}{N}\right]\right\}$ exists with a gap $g^{2}(\mu-v)^{2}$. The unique steady state is

$$
\widetilde{\Gamma}_{0}=-\frac{\mu^{2}-v^{2}}{\mu^{2}+v^{2}} \bigoplus_{n=1}^{N} i \sigma_{y}-\frac{2 \mu v}{\mu^{2}+v^{2}} \bigoplus_{n=1}^{N} i \sin (2 \pi n / N) \sigma_{x} .
$$

This state is a paired fermionic state according to the definition of Kraus et al. [35]. Paired states show two-particle quantum correlations that cannot be reproduced by separable states (mixtures of Slater determinants). In Ref. [35], it is proven that Gaussian states are paired iff $Q_{k l}=\left\langle\frac{i}{2}\left[a_{k}, a_{l}\right]\right\rangle \neq 0$. This condition expresses the fact that separable states are convex combinations of states with a fixed particle number. For the CM (52), we get

$$
Q_{k l}=\left\{\begin{array}{lll}
\frac{1}{2} \frac{\mu \nu \operatorname{sgn}(k-l)}{\mu^{2}+v^{2}}, & \text { if } & |k-l|=1, \\
0, & \text { if } & |k-l| \neq 1 .
\end{array}\right.
$$

We conclude that (50) generates paired states, except for the trivial cases $\mu=0$ or $v=0$. Note that, even though the gap closes at $\mu=v$ (where maximal pairing is created), there is no phase transition at this point.

\section{B. Noise-driven systems with Hamiltonians}

A different form of transitions can arise in the presence of a Hamiltonian when tuning the parameters of the Hamiltonian. To show this, we solve the evolution of the Lindblad master equation (16) with a general quadratic and translationally invariant Hamiltonian [see Eqs. (9) and (37)]. We choose 
the local Lindblad operators (47), again, because they are the simplest example. The diagonal master equation in Fourier space becomes

$$
\begin{aligned}
\partial_{t} \widetilde{\Gamma}= & {\left[\bigoplus_{n=1}^{N}\left(\begin{array}{cc}
i k_{n} & h_{n} \\
-h_{n}^{*} & i l_{n}
\end{array}\right), \widetilde{\Gamma}\right]-g^{2}\left(\mu^{2}+v^{2}\right) \widetilde{\Gamma} } \\
& -g^{2}\left(\mu^{2}-v^{2}\right) \bigoplus_{n=1}^{N} i \sigma_{y} .
\end{aligned}
$$

The corresponding steady-state $\mathrm{CM}$ in the weak-coupling limit $g \rightarrow 0$ is [36]

$$
\begin{aligned}
\widetilde{\Gamma}_{0}= & -\frac{\mu^{2}-v^{2}}{\mu^{2}+v^{2}} \bigoplus_{n=1}^{N} \frac{\operatorname{Re}\left(h_{n}\right)}{\left(k_{n}-l_{n}\right)^{2} / 4+\left|h_{n}\right|^{2}} \\
& \times\left(\begin{array}{cc}
i\left(k_{n}-l_{n}\right) / 2 & h_{n} \\
-h_{n}^{*} & -i\left(k_{n}-l_{n}\right) / 2
\end{array}\right) .
\end{aligned}
$$

Transforming back to $\Gamma_{0}$ [and using Eq. (38)], we can read off the particle number $\left\langle 2 a_{j}^{\dagger} a_{j}-1\right\rangle=\left(\Gamma_{0}\right)_{j j, 01}$ as

$$
\left(\Gamma_{0}\right)_{j j, 01}=\frac{1}{2} \frac{\mu^{2}-v^{2}}{\mu^{2}+v^{2}} \frac{1}{N} \sum_{n=1}^{N} \frac{\operatorname{Re}\left(h_{n}\right)^{2}}{\left(k_{n}-l_{n}\right)^{2} / 4+\left|h_{n}\right|^{2}} .
$$

Based on this result, we can now discuss how nonanalytic behavior in the steady state correlates with critical points of the system. A vanishing denominator in Eq. (56) is not $a$ priori a sufficient condition for nonanalytic behavior because the numerator might vanish at the same point. This is relevant for interesting examples with $k_{n}-l_{n}=0$, e.g., the $X Y$ chain in Eq. (45). We give a rigorous discussion in the following. In the thermodynamic limit, the sums over expectation values in Eq. (56) can be replaced by a loop integral around the origin of the complex plane with radius 1 where the integration variable is $z=\exp \left(\frac{2 \pi i}{N} n\right)$. This is possible because $h_{n}, k_{n}$, and $l_{n}$ are Fourier series. For local interactions, the denominator of the integrand is a polynomial in $z$ [see Eq. (35)] and, thus, has a finite number of distinct roots. Applying the residue theorem, a nonanalyticity in $\left\langle a_{j}^{\dagger} a_{j}\right\rangle$ is possible only if a residue of the integrand, i.e., a root of its denominator, moves through the integral contour in the complex plane as a function of some external parameters. This happens for a vanishing denominator $\left|h_{n}\right|^{2}+\left(k_{n}-l_{n}\right)^{2} / 4=0$ for some real $n \in[0, N)$. In the special case of a reflection symmetric system $k_{n}+l_{n}=0$, this coincides with a vanishing energy gap $\epsilon_{n}=0$ [see Eq. (40)], a signature for a quantum phase transition. To summarize, for a reflection symmetric system with $\left|h_{n}\right|^{2}+\left(k_{n}-l_{n}\right)^{2} / 4=0$ in the weak-coupling limit, a quantum phase transition occurs in the noise-driven system for the same parameter values as in the corresponding closed system and is signaled by a nonanalyticity in $\left\langle a_{j}^{\dagger} a_{j}\right\rangle$. This calculation is explicitly performed in Sec. VI A for the $X Y$ chain [37].

\section{QUADRATIC AND HERMITIAN LINDBLAD OPERATORS}

In this section, we turn to the dynamical properties of the Lindblad master equation with quadratic and Hermitian Lindblad operators as introduced in Sec. III B. In the study of closed systems, quantum phase transitions are signaled by nonanalyticities in ground-state expectation values. In the noise-driven case, the steady state is the analog of the ground state. However, in Sec. III B, we have shown that, in the case of Hermitian Lindblad operators, the steady states are trivial and, thus, cannot evidence a phase transition. Therefore, we turn to the ADR, which determines the long-time dynamics of the decoherence process. We identify nonanalytical behavior of this rate both in the absence of any Hamiltonian (see Sec. V A) for competing decoherence processes and for the nonzero Hamiltonian, in which case, phase transitions of the corresponding closed system are reflected in a dynamical transition of this rate (see Sec. V B).

\section{A. Purely noise-driven systems}

A particular simple set of local and quadratic Lindblad operators is

$$
\begin{aligned}
\mathbf{L}_{z}^{\alpha} & =g \mu \frac{i}{2}\left[c_{\alpha, 1}, c_{\alpha, 0}\right], \\
\mathbf{L}_{x}^{\alpha} & =g \nu \frac{i}{2}\left[c_{\alpha+1,0}, c_{\alpha, 1}\right] .
\end{aligned}
$$

In this case, the Lindblad equation (32) becomes

$$
\begin{aligned}
\partial_{t} \Gamma_{k l, u v}= & -4 g^{2} \mu^{2} \Gamma_{k l, u v}\left(1-\delta_{k l}\right) \\
& -4 g^{2} v^{2} \Gamma_{k l, u v}\left(1-\delta_{2 k+u+1,2 l+v} \delta_{k+1, l}\right. \\
& \left.-\delta_{2 k+u-1,2 l+v} \delta_{k-1, l}\right) .
\end{aligned}
$$

We can read off the decoherence rates $-4 g^{2}\left(\mu^{2}+v^{2}\right)$, $-4 g^{2} \mu^{2}$, and $-4 g^{2} v^{2}$. Thus, the ADR

$$
\Delta= \begin{cases}4 g^{2} \mu^{2}, & \text { if } \quad \mu \leqslant v, \\ 4 g^{2} v^{2}, & \text { if } \quad v<\mu\end{cases}
$$

undergoes a dynamical transition as a function of $\mu / v$ at $\mu=v$.

\section{B. Noise-driven systems with Hamiltonians}

Now, we add a quadratic Hamiltonian and calculate the asymptotic decay rate $\Delta$ in the limit of small couplings to the environment $g \rightarrow 0$. First, we derive it for the quadratic Lindblad operators from Eqs. (57) and (58) for $v=0$ and $\mu=1$. Later, we will present the results for the case of arbitrary $\mu$ and $v$. For translationally invariant systems, the Fourier transformed master equation (59) is

$$
\begin{aligned}
\partial_{t} \widetilde{\Gamma}_{k l} \equiv & (\widetilde{\mathcal{S}} \widetilde{\Gamma})_{k l}=[\widetilde{H}, \widetilde{\Gamma}]_{k l} \\
& -4 g^{2}\left(\widetilde{\Gamma}_{k l}-\frac{1}{N} \sum_{r, s=1}^{N} \widetilde{\Gamma}_{r s} \delta_{r-s, k-l}\right),
\end{aligned}
$$

with the unitarily transformed superoperator $\widetilde{\mathcal{S}}$ to

$$
\widetilde{\mathcal{S}}=(U \otimes U) \mathcal{S}(U \otimes U)^{\dagger},
$$

with $U$ from Eq. (35). For weak couplings between system and bath $g \rightarrow 0$, the eigenvalues of $\widetilde{\mathcal{S}}$ (and, thus, of $\mathcal{S}$ ) can be determined by first-order perturbation expansion. To this end, we first diagonalize the unperturbed Hamiltonian part of $\widetilde{\mathcal{S}}$,

$$
[\widetilde{H}, \widetilde{\Gamma}]_{k l}=\widetilde{H}_{k k} \widetilde{\Gamma}_{k l}-\widetilde{\Gamma}_{k l} \widetilde{H}_{l l} \stackrel{!}{=} \lambda \widetilde{\Gamma}_{k l},
$$


where we use the notation introduced in Eq. (36) for the Hamiltonian $\widetilde{H}$. The $4 N^{2}$ eigenvalues $\lambda^{m n a}(m, n=1, \ldots, N, a=$ $1, \ldots, 4)$ are

$$
\begin{aligned}
& \lambda^{m n 1}=i\left(\alpha_{m}-\alpha_{n}+\beta_{m}-\beta_{n}\right), \\
& \lambda^{m n 2}=i\left(\alpha_{m}-\alpha_{n}-\beta_{m}+\beta_{n}\right), \\
& \lambda^{m n 3}=i\left(\alpha_{m}-\alpha_{n}+\beta_{m}+\beta_{n}\right), \\
& \lambda^{m n 4}=i\left(\alpha_{m}-\alpha_{n}-\beta_{m}-\beta_{n}\right),
\end{aligned}
$$

with

$$
\begin{gathered}
\alpha_{m}=\left|k_{m}+l_{m}\right| / 2, \\
\beta_{m}=\sqrt{\left|h_{m}\right|^{2}+\left(k_{m}-l_{m}\right)^{2} / 4} .
\end{gathered}
$$

The corresponding eigenmatrices are denoted as $\widetilde{\Lambda}^{m n a}$ with nonzero elements $\widetilde{\Lambda}_{k l}^{m n a}$ only for $m=k$ and $n=l$, i.e., $\widetilde{\Lambda}_{k l}^{m n a}=$ $\delta_{m k} \delta_{n l} \widetilde{\Lambda}_{m n}^{m n a}$. Perturbation theory demands calculating the matrix elements of the perturbative part of $\widetilde{\mathcal{S}},-4 g^{2}\left(\delta_{m k} \delta_{n l} \delta_{a b}-\right.$ $\left.P_{k l b}^{m n a} / N\right)$ [see Eq. (61)], with

$$
\begin{aligned}
P_{k l b}^{m n a} & =\frac{N}{4 g^{2}}\left\langle\widetilde{\Lambda}^{m n a}\left|\frac{1}{2} \sum_{\alpha}\left(\mathcal{L}^{\alpha}\right)^{2}\right| \widetilde{\Lambda}^{k l b}\right\rangle+N \delta_{m k} \delta_{n l} \delta_{a b} \\
& =\sum_{q, r, s, t=1}^{N} \delta_{q-r, s-t} \operatorname{Tr}\left[\left(\widetilde{\Lambda}_{s t}^{m n a}\right)^{\dagger} \widetilde{\Lambda}_{q r}^{k l b}\right] \\
& =\delta_{m-n, k-l} \operatorname{Tr}\left[\left(\widetilde{\Lambda}_{m n}^{m n a}\right)^{\dagger} \widetilde{\Lambda}_{k l}^{k l b}\right] .
\end{aligned}
$$

Thus, the eigenvalues of $\widetilde{\mathcal{S}}$ are determined by those of the Hermitian matrix $P$, and the largest eigenvalue smaller than $N$ of $P$ (restricted to a space of degenerate eigenvalues $\lambda^{m n a}$ of $[\tilde{H}, \cdot])$ determines the ADR. We denote it by $\Delta_{P}$ and, thus, have that the asymptotic rate is $\Delta=4 g^{2}\left(1-\frac{\Delta_{P}}{N}\right)$. To find $\Delta_{P}$, note that the matrix elements of $P$ fulfill $\left|P_{k l b}^{m n a}\right| \leqslant 1$. Thus, an $N$-fold degeneracy of $\lambda^{m n a}$ is required for $\Delta_{P}=\Omega(N)$. Generically, this is possible only for the eigenvalue $\lambda^{m n a}=0$, i.e., $m=n$ and $a=1,2$. The corresponding eigenmatrices are

$$
\begin{gathered}
\widetilde{\Lambda}_{k l}^{m m 1}=\delta_{m k} \delta_{m l} \frac{1}{\sqrt{2} \beta_{n}}\left(\begin{array}{cc}
i \frac{k_{m}-l_{m}}{2} & -h_{m} \\
h_{m}^{*} & -i \frac{k_{m}-l_{m}}{2}
\end{array}\right), \\
\widetilde{\Lambda}_{k l}^{m m 2}=\delta_{m k} \delta_{m l} \frac{1}{\sqrt{2}}\left(\begin{array}{cc}
1 & 0 \\
0 & 1
\end{array}\right) .
\end{gathered}
$$

As the eigenmatrices $\widetilde{\Lambda}^{m m 2}$ give eigenvalues equal to $N$ and 0 only, have no overlap with physical CMs, and yield $P_{k l 1}^{m n 2}=0$, we focus on the matrices $\widetilde{\Lambda}^{m m 1}$. The corresponding part of the perturbation matrix is

$$
P_{m n}=P_{n n 1}^{m m 1}=\frac{2 h_{m} h_{n}^{*}+2 h_{m}^{*} h_{n}-\left(k_{m}-l_{m}\right)\left(k_{n}-l_{n}\right)}{4 \beta_{m} \beta_{n}} .
$$

We diagonalize this matrix by introducing the three vectors $|a\rangle,|b\rangle,|c\rangle \in \mathbb{C}^{N}$ with the components,

$$
a_{m}=\frac{k_{m}-l_{m}}{2 \beta_{m}}, \quad b_{m}=\frac{\operatorname{Im}\left(h_{m}\right)}{\beta_{m}}, \quad c_{m}=\frac{\operatorname{Re}\left(h_{m}\right)}{\beta_{m}},
$$

and writing $P_{m n}$ in terms of these un-normalized vectors,

$$
P=|c\rangle\langle c|+| b\rangle\langle b|-| a\rangle\langle a| .
$$

We now exploit the symmetries of $h_{n}, k_{n}$, and $l_{n}$ stated in Eq. (38). First, we observe that $|c\rangle$ is orthogonal to $|a\rangle$ and $|b\rangle$. We have chosen the CMs corresponding to the three vectors (74) to be anti-Hermitian since this matrix remains anti-Hermitian even in the complex vector space. After transforming back into real space, the ones corresponding to $|a\rangle$ and $|b\rangle$ are purely imaginary so that they have no overlap with any physically meaningful real and antisymmetric CM. Only the matrix corresponding to $|c\rangle$ is real and antisymmetric and is given by

$$
\Gamma_{\Delta}=\left(\sum_{m=1}^{N} \frac{\left|h_{m}\right|^{2}}{2 \beta_{m}^{2}}\right)^{-1} \sum_{n} \frac{\operatorname{Re}\left(h_{n}\right)}{\beta_{n}} U^{\dagger} \Lambda^{n n 1} U .
$$

Therefore, it determines the ADR. We get

$$
\Delta_{P}=\sum_{m=1}^{N} \frac{\operatorname{Re}\left(h_{m}\right)^{2}}{\beta_{m}^{2}}=\sum_{m=1}^{N} \frac{\operatorname{Re}\left(h_{m}\right)^{2}}{\left|h_{m}\right|^{2}+\left(k_{m}-l_{m}\right)^{2} / 4},
$$

and thus,

$$
\Delta=\frac{4 g^{2}}{N} \sum_{m=1}^{N} \frac{4 \operatorname{Im}\left(h_{m}\right)^{2}+\left(k_{m}-l_{m}\right)^{2}}{4\left|h_{m}\right|^{2}+\left(k_{m}-l_{m}\right)^{2}}
$$

as the general form of the ADR.

We can extend our analysis to systems with the general Lindblad operators Eqs. (57) and (58) and find, in an analogous way, the two lowest decay rates,

$$
\frac{\Delta_{ \pm}}{4 g^{2}}=\mu^{2}+v^{2}-\frac{\epsilon_{z}+\epsilon_{x}}{2} \pm \sqrt{\left(\frac{\epsilon_{z}-\epsilon_{x}}{2}\right)^{2}+\epsilon^{2}},
$$

with

$$
\begin{aligned}
\epsilon_{z} & =\frac{\mu^{2}}{N} \sum_{m=1}^{N} \frac{\operatorname{Re}\left(h_{m}\right)^{2}}{\beta_{m}^{2}}, \\
\epsilon_{x} & =\frac{\nu^{2}}{N} \sum_{m=1}^{N} \frac{\operatorname{Re}\left[h_{m} \exp (-2 \pi i m / N)\right]^{2}}{\beta_{m}^{2}}, \\
\epsilon & =\frac{\mu \nu}{N} \sum_{m=1}^{N} \frac{\operatorname{Re}\left(h_{m}\right) \operatorname{Re}\left[h_{m} \exp (-2 \pi i m / N)\right]}{\beta_{m}^{2}} .
\end{aligned}
$$

We can now argue that the ADR itself reflects the criticality of the system. The argument is completely analogous to the one given in Sec. IV B. If the denominator becomes zero, we can expect nonanalyticity expressions Eqs. (80). In particular, in the reflection symmetric case $k_{n}+l_{n}=0$ where the denominator agrees with the elementary excitation energies $\left[\epsilon_{n}^{2}=\left|h_{n}\right|^{2}+\left(k_{n}-l_{n}\right)^{2} / 4=0\right.$, see Eq. (40)], the nonanalyticity in the ADR signals the presence of a quantum phase transition in the Hamiltonian itself.

\section{EXAMPLE HAMILTONIANS}

In this section, we will revisit the results obtained for the steady state and the ADR for linear and quadratic Lindblad operators in Secs. IV B and VB for the specific Hamiltonian (12) of the quantum $X Y$ chain.

The energies of the elementary excitations of this Hamiltonian are $\epsilon_{n}=\left|h_{n}\right|$. Thus, for the $X Y$ chain in Eq. (45), the 
gap closes at $B=2 J$ in the thermodynamic limit, and the quantum $X Y$ chains exhibit a phase transition at this point. In fact, these models constitute the archetypal example of a continuous quantum phase transition [18]. In this section, we want to find properties of the noise-driven dynamics signaling this phase transition.

\section{A. Linear Lindblad operators}

Let us now apply the findings from Sec. IV B and Eq. (56) to the example system defined in Eq. (45), which contains a quantum phase transition at $B=2 J$. Then, the particle numbers become, for $k_{n}=l_{n}=0$,

$$
\left\langle 2 a_{n}^{\dagger} a_{n}-1\right\rangle=\frac{1}{2} \frac{\mu^{2}-v^{2}}{\mu^{2}+v^{2}}\left(1+\frac{1}{N} \sum_{n=1}^{N} \frac{h_{n}^{*}}{h_{n}}\right) .
$$

For $\gamma=0$, we easily obtain $\Delta=0$ [since, by Eq. (45), $h_{n}$ is real in that case and then, by Eq. (78), $\Delta$ is zero for $k_{n}=l_{n}=$ 0]. For $\gamma \neq 0$, we evaluate the sum $1 / N \times \sum_{m=1}^{N} h_{m}^{*} / h_{m}$ in the thermodynamic limit by introducing the complex variable $z=\exp (-2 \pi i m / N)$,

$$
\begin{aligned}
& \lim _{N \rightarrow \infty} \frac{1}{N} \sum_{m=1}^{N} \frac{h_{m}^{*}}{h_{m}} \\
& \quad=\frac{1}{2 \pi i} \oint_{|z|=1} \frac{d z}{z} \frac{2 J(1-\gamma) z^{2}-2 B z+2 J(1+\gamma)}{2 J(1+\gamma) z^{2}-2 B z+2 J(1-\gamma)},
\end{aligned}
$$

where the integration contour is a circle of radius $|z|=1$ around $z=0$ in the complex plane. The complex integrand is analytic except for three distinct poles at

$$
z^{0}=0, \quad z^{ \pm}=\frac{1}{2 J(1+\gamma)}\left[B \pm \sqrt{B^{2}-4 J^{2}\left(1-\gamma^{2}\right)}\right] .
$$

The contour integral is determined by the sum over the residues at those poles, which are inside the contour $(|z|<$ 1). $z^{0}$ is always inside this contour. In the case of $\gamma>0, z^{+}$is inside the contour for $0 \leqslant B<2 J$ and is outside for $B>2 J$, whereas, $z^{-}$is always inside the contour. In the case of $\gamma<$ $0, z^{-}$is inside the contour for $B>2 J$ and is outside for $0 \leqslant$ $B<2 J$, whereas, $z^{+}$is always outside the contour. So residues cross the contour at the quantum phase transition $B=2 \mathrm{~J}$ (because, then, $h_{n}=0$ for some $n$ ), leading to a nonanalytical behavior in the particle density of the steady state (see Fig. 1).

After applying the residue theorem, we get the particle number of the steady state,

$$
\begin{aligned}
&\left\langle 2 a_{n}^{\dagger} a_{n}-1\right\rangle \\
&=\frac{\mu^{2}-v^{2}}{\mu^{2}+v^{2}} \begin{cases}\frac{1}{1+|\gamma|}, & B \leqslant 2 J, \\
\frac{1}{1-\gamma^{2}}\left(1-\frac{\gamma^{2}}{\sqrt{1-\left(\frac{2 J}{B}\right)^{2}\left(1-\gamma^{2}\right)}}\right), & B \geqslant 2 J\end{cases}
\end{aligned}
$$

for all $\gamma$, which does not depend on the sign of $\gamma$. For $B<2 J$, the particle number in the steady state does not vary with the magnetic field, whereas, its magnitude approaches $\left(\mu^{2}-v^{2}\right) /\left(\mu^{2}+v^{2}\right)$ for large magnetic fields $\sim(J / B)^{2}$. To summarize, the steady state undergoes a noise-driven phase transition at $B=2 J$ signaling the phase transition in the system.

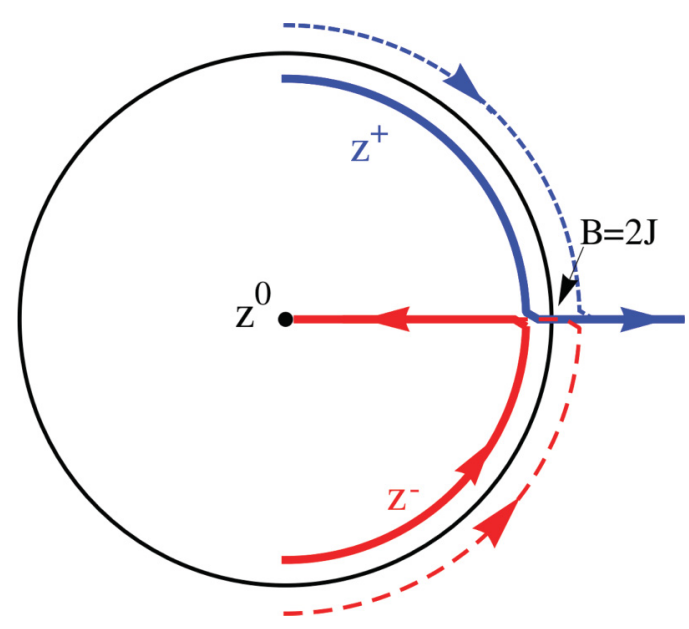

FIG. 1. (Color online) The poles $z^{0}, z^{ \pm}$[see Eq. (83)] are plotted for $J=1, \gamma= \pm 0.1$. As $B$ is changed from 0 to 20 , the poles $z^{+}\left(z^{-}\right)$ for positive anisotropy $\gamma=+0.1$ move along the blue (red) solid curves, and $z^{+}$crosses the contour at the critical value $B=2 \mathrm{~J}$. For negative $\gamma=-0.1, z^{-}$crosses at $B=2 J$. At the crossing, the integral Eq. (82) changes nonanalytically.

\section{B. Quadratic and Hermitian Lindblad operators}

As an example, we study the anisotropic $X Y$ chain in a transverse magnetic field with the Hamiltonian given in Eq. (12). This translationally invariant Hamiltonian is JordanWigner transformed to a quadratic fermionic Hamiltonian with Hamiltonian matrix $H$ given by

$$
\begin{gathered}
H_{0}=\left(\begin{array}{cc}
0 & -2 B \\
2 B & 0
\end{array}\right), \\
H_{1}=\left(\begin{array}{cc}
0 & 2 J(1-\gamma) \\
-2 J(1+\gamma) & 0
\end{array}\right), \\
H_{-1}=\left(\begin{array}{cc}
0 & 2 J(1+\gamma) \\
-2 J(1-\gamma) & 0
\end{array}\right) .
\end{gathered}
$$

After Fourier transforming [see Eq. (35)], this Hamiltonian matrix assumes the form given in Eq. (37) with parameters $h_{n}, k_{n}, l_{n}$ given by Eq. (45).

We now apply the results from Sec. V B to the Hamiltonian Eq. (85) and the Lindblad operators,

$$
\mathbf{L}^{\alpha}=g \mu \frac{i}{2}\left[c_{\alpha, 1}, c_{\alpha, 0}\right] \leftrightarrow g \sigma_{z}^{\alpha} .
$$

After a brief discussion of the steady states and a derivation of the ADR in the thermodynamic $(N \rightarrow \infty)$ and in weak coupling $(g \rightarrow 0)$ limits, we present numerical results of the system dynamics for finite $N$ and $g$ and compare them with our analytic predictions.

First, we discuss the steady states of these systems (see Sec. III B). From Eq. (31), we have concluded that the steadystate density matrix is the identity up to symmetries shared by the Lindblad operators and the Hamiltonian. A rigorous derivation of the steady states for this example could start from the ansatz that the steady-state density matrix is diagonal in the Fock basis, following from $\left[\sigma_{z}^{\alpha}, \rho\right]=0$. Then, the commutator $[\mathbf{H}, \rho]=0$ must be exploited to get the steady state.

As the Lindblad operators correspond to local particle number operators, the important compatible symmetries for 
the $X Y$ chains are the parity $\mathcal{P}=\sigma_{z}^{1} \cdots \sigma_{z}^{N}$, discriminating between an odd and an even number of particles, and the total particle number $\mathcal{N}=\left(\mathbf{1}+\sum \sigma_{z}^{j}\right) / 2$. For truly asymmetric $X Y$ chains $\gamma \neq 0.5$, the parity is the highest symmetry compatible with the Lindblad operators. In these cases, the steady-state density matrix is given by the identity in the two sectors of even and odd parities; the relative weight of these sectors is determined by the initial state. For the symmetric chain $\gamma=0.5$, the steady-state density matrix is the identity only in the sectors with a constant total number of particles. Thus, for $\gamma \neq 0.5$, the steady-state magnetization is $\left\langle\sigma_{z}^{j}\right\rangle=0$ regardless of the initial state, whereas, the magnetization of the initial state is conserved for $\gamma=0.5$.

Second, we calculate the ADR (78) for the $X Y$ chains with Eq. (45) analogous to the integration in Sec. VI A.

After applying the residue theorem, we get the ADR,

$$
\Delta=4 g^{2} \begin{cases}\frac{|\gamma|}{1+|\gamma|}, & B \leqslant 2 J, \\ \frac{\gamma^{2}}{1-\gamma^{2}}\left\{\left[1-\left(\frac{2 J}{B}\right)^{2}\left(1-\gamma^{2}\right)\right]^{-1 / 2}-1\right\}, & B \geqslant 2 J\end{cases}
$$

for all $\gamma$ in the case of $\mu=1$. It does not depend on the sign of $\gamma$ and is shown in Fig. 2 for several values of $\gamma \in[0,1]$. For $B<2 J$, the ADR does not vary with the magnetic field, whereas for large magnetic fields its magnitude decreases to zero and scales as $(J / B)^{2}$. The same behavior was found for the variance in the particle number in these models in a previous paper [38]. To summarize, the ADR undergoes a noise-driven phase transition at $B=2 J$ signaling the phase transition in the system.

The final result for the ADR (89) is valid in the limits $N \rightarrow \infty$ and $g \rightarrow 0$. In this section, we perform a numerical diagonalization of the Lindblad master equation superoperator $\mathcal{S}$ to compare the analytic result with the values for finite $N$ and $g$. Furthermore, we extract the ADR from a simulation of the system dynamics and compare it with our prediction.

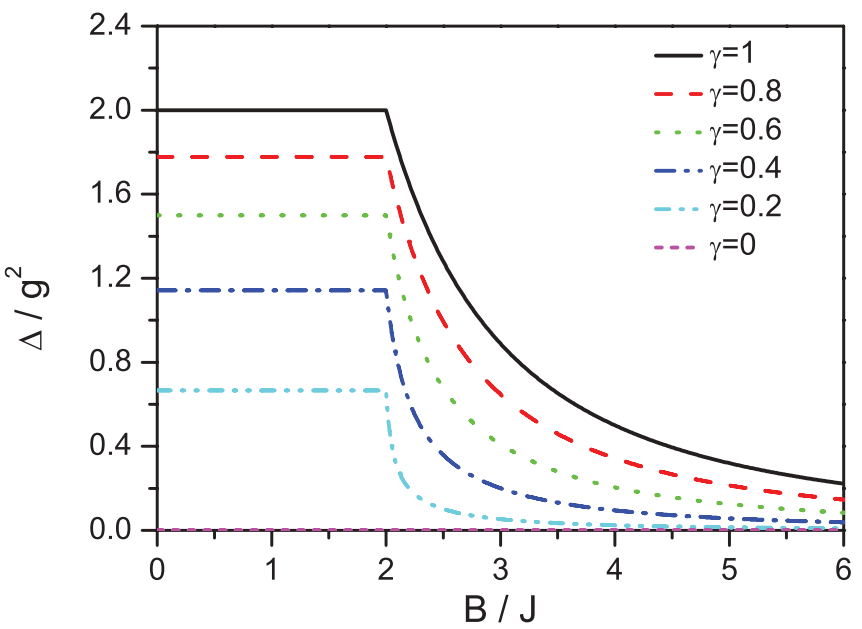

FIG. 2. (Color online) Asymptotic decay rate $\Delta$ [see Eq. (78)] of the $X Y$ chain (12) for different anisotropy parameters $\gamma$ as a function of the magnetic field in the limits $N \rightarrow \infty$ and $g \rightarrow 0$. A phase transition in $\Delta$ is visible at $B=2 J$ for $\gamma \neq 0$.

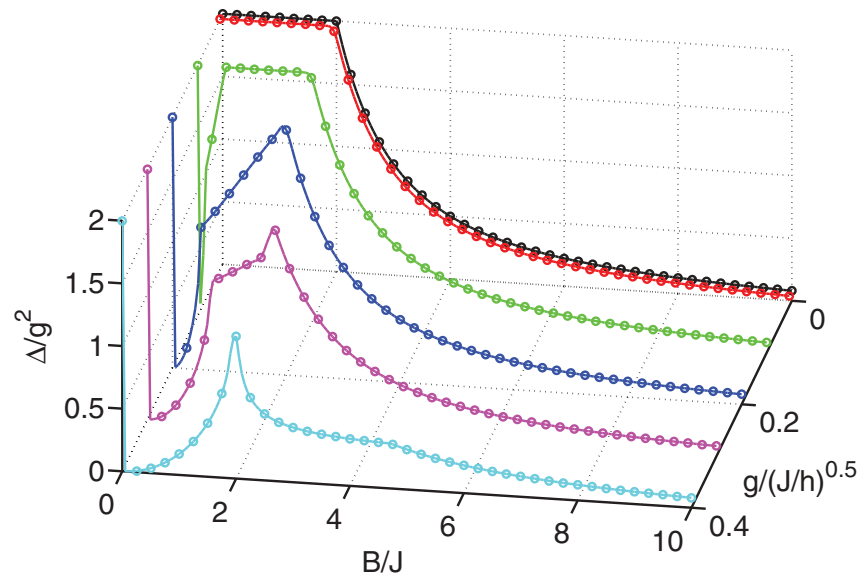

FIG. 3. (Color online) Asymptotic decay rate $\Delta$ [see Eq. (78)] of the $X Y$ chain (12) for different coupling strengths $g, \gamma=1$, and $N=100$ as a function of the magnetic field $B$. For $g \leqslant 0.1(J / \hbar)^{0.5}$, the results agree with the limit of weak coupling $g \rightarrow 0$ [see Eq. (89)].

In Fig. 3, we present the ADR for finite coupling strengths $g$. For $g^{2} \leqslant 0.01 J / \hbar$, the result of perturbation theory is in excellent agreement with the numerical diagonalization of the Lindblad master equation superoperator. Deviations are strongest at small magnetic fields for which the finite $g$ is no longer a small perturbation. The nonanalytic behavior at the critical field value $B / J=2$ is clearly visible. The additional structure in the ADR for finite $g$ and small $B / J$ arises from level crossings in the spectrum of the Liouvillian. At $B=0$, the steady state becomes highly degenerate. The ADR (the largest nonzero real part) jumps to a finite value indicating a finite gap above the steady-state manifold.

We show the ADR $\Delta$ for different (finite) system sizes in Fig. 4. Even in small systems with $N=10$ spins, the same qualitative behavior is found as in thermodynamic limit, i.e., the ADR signals the quantum phase transition in the system at

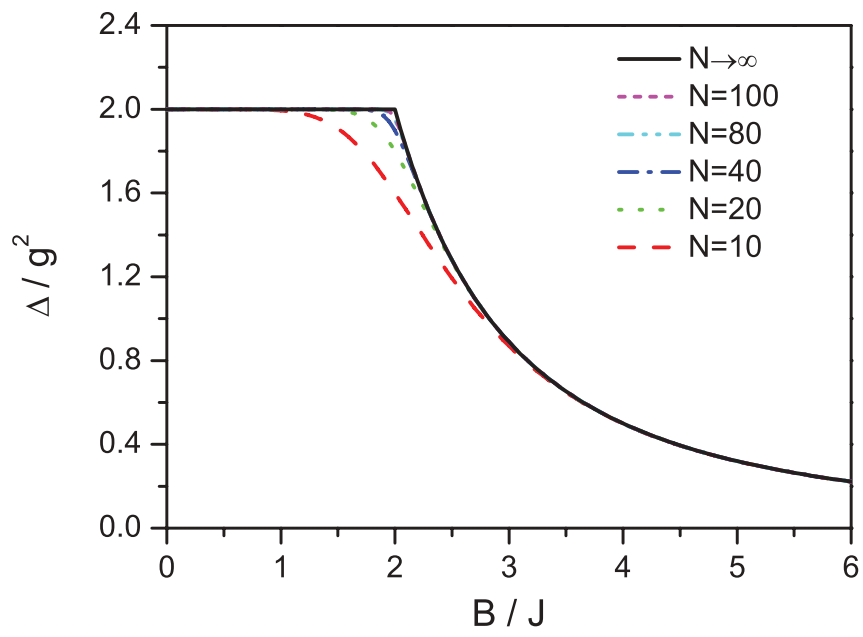

FIG. 4. (Color online) Asymptotic decay rate $\Delta$ [see Eq. (89)] of the $X Y$ chain (12) for different system sizes $N$ and $\gamma=1, g=$ $0.01(J / \hbar)^{0.5}$ as a function of the magnetic field $B$. For $N \geqslant 50$, the thermodynamic limit is reached, except for small variations at the phase transition $B=2 J$. 


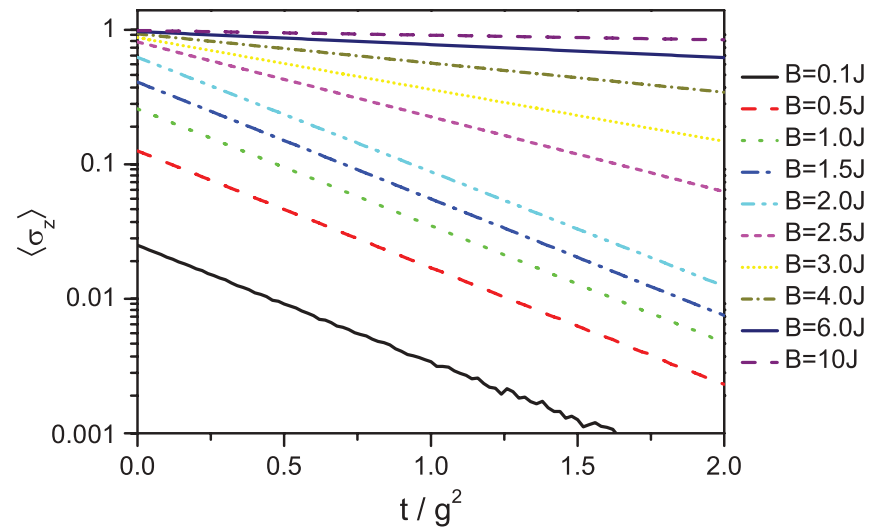

FIG. 5. (Color online) Evolution of the magnetization $\left\langle\sigma_{z}^{j}\right\rangle$ in time starting from the system ground state of the $X Y$ chain (12) for different magnetic fields $B, g=0.01(J / \hbar)^{0.5}$, and $\gamma=1$. The magnetization decreases exponentially in time.

$B=2 J$. However, finite values of $g$ and $N$ lead to a smearing out of the phase transition.

We have defined the ADR through a diagonalization of the master equation, trying to describe the long-time dynamics of the system. To demonstrate the deep relation between $\Delta$ and the noise-driven dynamics, we extract the decoherence rate from a dynamical calculation (see Fig. 5). Here, we start from the ground state of the system and study the decay of the magnetization in time after the system is brought into contact with a Markovian bath. In this example, the exponential decay expected after long evolution times is nicely visible. In Fig. 6, we compare the extracted decay rates for different magnetic fields with the result of the diagonalization. We find an exact agreement with the ADR numerically calculated with the same finite parameters.

We can calculate the ADR for the $X Y$ chain for general values of $\mu$ and $v$ in a similar way. In the spin picture, the

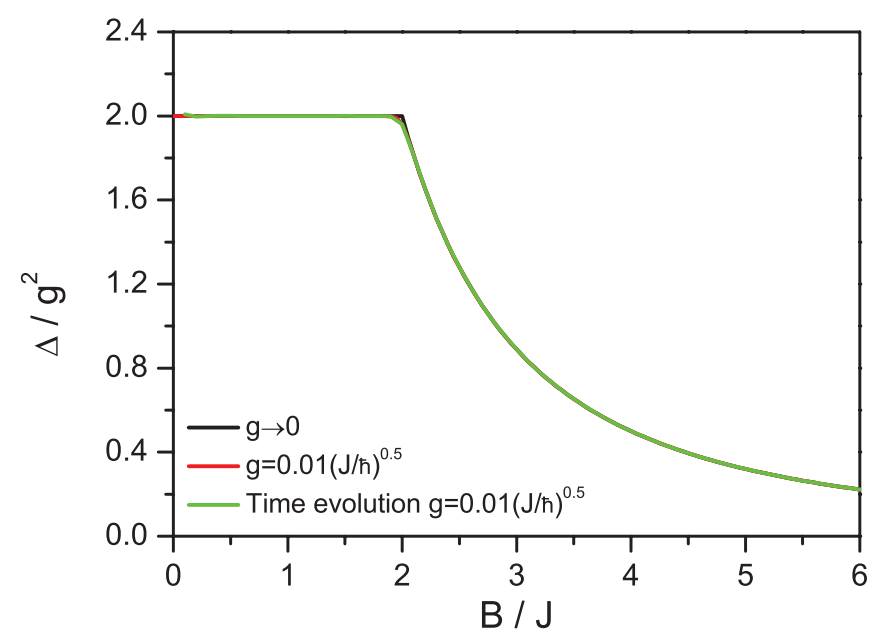

FIG. 6. (Color online) The ADRs $\Delta$ [see Eq. (89)] of the $X Y$ chain (12) for $\gamma=1$, for $g=0.01(J / \hbar)^{0.5}$, and for $g \rightarrow 0$ (result of perturbation theory) as a function of the magnetic field $B$ are compared with the late-time decoherence rates extracted from Fig. 5. The agreement between the ADR and the late-time decoherence rate shows the validity of our calculations for finite times.
Lindblad operators are

$$
\begin{gathered}
\mathbf{L}_{z}^{\alpha}=g \mu \sigma_{\alpha}^{z}=g \mu \frac{i}{2}\left[c_{\alpha, 1}, c_{\alpha, 0}\right], \\
\mathbf{L}_{x}^{\alpha}=g \nu \sigma_{\alpha}^{x} \sigma_{\alpha+1}^{x}=g \nu \frac{i}{2}\left[c_{\alpha, 0}, c_{\alpha+1,1}\right] .
\end{gathered}
$$

We find for the constants in Eq. (79) in the case $\gamma=1$,

$$
\begin{gathered}
\epsilon_{z} / \mu^{2}= \begin{cases}\frac{1}{2}, & B \leqslant 2 J, \\
1-\frac{1}{2}\left(\frac{2 J}{B}\right)^{2}, & B \geqslant 2 J,\end{cases} \\
\epsilon_{x} / \mu^{2}= \begin{cases}1-\frac{1}{2}\left(\frac{B}{2 J}\right)^{2}, & B \leqslant 2 J, \\
\frac{1}{2}, & B \geqslant 2 J,\end{cases} \\
\epsilon /(\mu \nu)= \begin{cases}-\frac{B}{2 J}, & B \leqslant 2 J, \\
-\frac{2 J}{B}, & B \geqslant 2 J .\end{cases}
\end{gathered}
$$

In the symmetric case $\mu=v$, the $\operatorname{ADR}$ is constant $(\Delta=$ $\left.-4 g^{2} \mu^{2}\right)$. However, the next larger decoherence rate changes nonanalytically,

$$
\Lambda_{-}= \begin{cases}-2 g^{2} \mu^{2}\left[3+\left(\frac{B}{2 J}\right)^{2}\right], & \text { if } B<2 J \\ -2 g^{2} \mu^{2}\left[3+\left(\frac{2 J}{B}\right)^{2}\right], & \text { if } B>2 J\end{cases}
$$

\section{EXPERIMENTAL REALIZATION}

We now discuss an experiment suited for the measurement of the asymptotic decoherence rate in spin systems. The quantum simulation of spin systems with trapped ions was proposed in Ref. [39] where the spin degree of freedom is represented by two hyperfine levels. The magnetic field can be simulated either by directly driving Rabi oscillations of the hyperfine transition or with position-independent Raman transitions induced by suitably aligned lasers. The spin-spin interaction is mediated via motional degrees of freedoms. State-dependent optical dipole forces (compare with state-dependent optical lattices) are generated by coupling the two hyperfine levels to electronically excited states with off-resonant laser beams. These dipole forces change the distance and, consequently, the Coulomb repulsion between two ions dependent on their internal states. This state-dependent Coulomb repulsion can be designed to give the required spin-spin interaction. The spin state can be measured by fluorescence imaging of the ions.

In this way, the quantum Ising chain $[40,41]$ and frustrated Ising models [42] have been realized in recent experiments. In these experiments, the ions were first cooled to their zero-point motional ground state and optically pumped into a certain spin configuration representing the ground state of the system without spin-spin interactions. Then, the spin-spin interactions were adiabatically increased such that the system underwent a phase transition. Finally, it was checked that the final state represented the ground state of the simulated Hamiltonian. A large noncritical two-dimensional Ising system has been simulated with ions in a Penning trap [43]. In the digital approach to quantum simulation with trapped ions, the elements of a general toolbox, including Hamiltonian and noise-driven dynamics, have been demonstrated [44,45].

In the following, we describe how to extend analog quantum simulation to include an incoherent evolution. The Lindblad master equation (3) with Hermitian Lindblad operators $\mathbf{L}^{\alpha}=g \sigma_{z}^{\alpha}$ (see Sec. VB) can be realized by introducing fluctuations in the simulated magnetic field 
$B^{\alpha}(t)=B^{\alpha}+\delta B^{\alpha}(t)$ [46] as shown in the following. The local magnetic fields $\delta B^{\alpha}(t)$ should be uncorrelated between different sites $\overline{\delta B^{\alpha}\left(t_{1}\right) \delta B^{\beta}\left(t_{2}\right)}=\delta_{\alpha \beta} \overline{\delta B^{\alpha}\left(t_{1}\right) \delta B^{\alpha}\left(t_{2}\right)}$. We restrict our derivation to a single Lindblad operator without loss of generality. Let, for example, $\delta B(t)$ constitute a Gaussian stochastic process of zero mean $\overline{\delta B(t)}=0$ with the time correlations,

$$
\overline{\delta B\left(t_{1}\right) \delta B\left(t_{2}\right)}=\frac{\overline{\delta B^{2}}}{\sqrt{2 \pi}} \exp \left[-\frac{\left(t_{1}-t_{2}\right)^{2}}{2 T^{2}}\right] .
$$

The correlation time $T$ has to be much shorter than every process in the system (Markovian limit), i.e., $\|\mathcal{H}\| T<\omega T \ll 1$ with the spectral width $\omega$ of the Hamiltonian (difference between largest and smallest eigenvalues) and the superoperator $\mathcal{H}$ from Eq. (26). The averaged density matrix evolves like $|\rho(t)\rangle=\overline{\mathcal{U}(t)}|\rho(0)\rangle$ where the bar denotes the statistical average over the fluctuating magnetic field. The time-evolution operator $\overline{\mathcal{U}(t)}$ consists of contributions from $\mathcal{H}$ and

$$
\mathcal{V}(t)=\frac{\delta B(t)}{\hbar} \mathcal{V}=-\frac{i \delta B(t)}{\hbar}\left(\mathbf{V} \otimes \mathbf{1}-1 \otimes \mathbf{V}^{T}\right),
$$

with $\mathbf{V}=\sigma_{z}$. We can evaluate the statistical average of the time-evolution operator in the interaction picture for the superoperators,

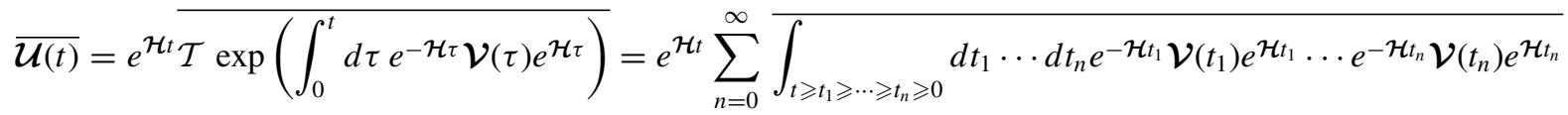

$$
\begin{aligned}
& =e^{\mathcal{H} t} \sum_{m=0}^{\infty}\left(\frac{1}{\hbar^{2}} \int_{0}^{\infty} \overline{\delta B(0) \delta B(\tau)} d \tau\right)^{m} \times \int_{t \geqslant t_{1} \geqslant \cdots \geqslant t_{m} \geqslant 0} d t_{1} \cdots d t_{m} e^{-\mathcal{H} t_{1}} \mathcal{V}^{2} e^{\mathcal{H} t_{1}} \cdots e^{-\mathcal{H} t_{m}} \mathcal{V}^{2} e^{\mathcal{H} t_{m}} \\
& =e^{\mathcal{H} t} \sum_{m=0}^{\infty}\left(\frac{\overline{\delta B^{2}}}{\hbar^{2}} \times \frac{T}{2}\right)^{m} \times \int_{t \geqslant t_{1} \geqslant \cdots \geqslant t_{m} \geqslant 0} d t_{1} \cdots d t_{m} e^{-\mathcal{H} t_{1}} \mathcal{V}^{2} e^{\mathcal{H} t_{1}} \cdots e^{-\mathcal{H} t_{m}} \mathcal{V}^{2} e^{\mathcal{H} t_{m}} \\
& \overline{\mathcal{U}(t)}=\exp \left(\mathcal{H} t+\frac{1}{2} \frac{\overline{\delta B^{2}} T}{\hbar^{2}} \mathcal{V}^{2} t\right)
\end{aligned}
$$

with the time-ordering operator $\mathcal{T}$. Between the second and the third lines, we keep only even summation indices $m=2 n$ (zero-mean Gaussian process), evaluate the statistical average at adjacent times $t_{2 n-1}-t_{2 n} \leqslant T$ (correlation time $T$; that only adjacent times need to be considered is a consequence of time ordering, the Gaussian factorization of higher-order correlations, and the very short correlation times), and neglect the terms $\exp \left[\mathcal{H}\left(t_{2 n-1}-t_{2 n}\right)\right] \ll 1$ (Markovian limit). To summarize, we have shown that the described fluctuations of the magnetic field generate Markovian dynamics [see Eq. (25)] with Lindblad operators $\mathbf{L}^{\alpha}=g \sigma_{z}^{\alpha}=g \mathbf{V}$ and decoherence strength,

$$
g^{2}=\frac{\overline{\delta B^{2}} T}{\hbar^{2}}
$$

In the case of the anisotropic $X Y$ chain [see Eq. (12)], the correlation time $T$ is bounded by the width of the single-particle excitation spectrum $T^{-1} \gg \max (4 B / \hbar, 8 J / \hbar)$. In the recent experiment [40], $2 J / \hbar \approx B / \hbar=2 \pi \times 4.4 \mathrm{kHz}$ was used, but experimentally available laser intensities allow $2 J / \hbar \approx$ $B / \hbar \approx 2 \pi \times 40 \mathrm{kHz}$. We propose creating fluctuations in the magnetic field with frequency $T^{-1}=2 \pi \times 1.6 \mathrm{MHz}$ and variance $\overline{\delta B^{2}} / \hbar^{2}=(0.2 B / \hbar)^{2} \approx(2 \pi \times 8 \mathrm{kHz})^{2}$. This would result in the decoherence strength $g^{2} \approx 2 \times 10^{-3} \mathrm{~J} / \hbar$ and would require coherence times on the order of $2 \pi / g^{2} \approx 25 \mathrm{~ms}$. These coherence times can, in principle, be achieved in systems of trapped ions [47].

\section{CONCLUSION}

We have investigated the dynamics of noise-driven open quantum systems with regard to their steady states and asymptotic decay. We have shown that insight into different phases can be gained by spectral analysis of the Liouvillian in analogy to how the spectrum of the Hamiltonian reveals critical behavior in zero-temperature quantum phase transitions.

To illustrate this point, we have analyzed, in detail, the Liouvillian of fermionic systems under a translationally invariant quadratic Hamiltonian, coupled to a Markovian bath. We treat master equations with linear or quadratic and Hermitian Lindblad operators. In both cases, the master equation leads to a closed equation for the CM from which the steady-state $\mathrm{CM}$ and the rates at which it is approached can be obtained exactly (see also Ref. [19] for an elegant and comprehensive treatment of both fermionic and bosonic linear open systems and their critical properties and Ref. [28] for a detailed study of transport in spin chains under dissipation and dephasing). These results apply as well to a large class of 1D spin systems that can be mapped to quasifree fermions by a Jordan-Wigner transformation. We have proposed an experimental realization of this quantum simulation with trapped ions. Numerical calculations show that our results for the weak decoherence limit do apply to such finite systems.

We have focused on the limit of weak decoherence $(g \rightarrow 0)$ and have shown how to deduce information about critical points from the spectrum of the Liouvillian. In particular, the $\operatorname{ADR} \Delta$, i.e., the smallest nonzero eigenvalue of the Liouvillian, can serve as an indicator of phase transitions even if the steady state of the system is trivial and steady-state expectation values, thus, cannot yield such information (as in the case of Hermitian Lindblad operators). Depending on the decoherence process considered, the critical point can be reflected in the spectrum of the system's Liouvillian in 
TABLE I. Different noise-driven systems studied, characterized by their Lindblad operators and Hamiltonian $H$. Relevant properties of the ADR $\Delta$ and the steady state are listed. $x_{c}$ denotes critical points of the Hamiltonian $H$.

\begin{tabular}{lcc}
\hline \hline Lindblad operator & ADR and gap & Steady state \\
\hline$\mu a_{\alpha}, v a_{\alpha}^{\dagger}$ & Hamiltonian $H=0$ & Thermal \\
$\mu a_{\alpha}+v a_{\alpha+1}^{\dagger}$ & Gapped, no phase transition & Paired \\
$\frac{i \mu}{2}\left[c_{\alpha, 0}, c_{\alpha, 1}\right], \frac{i v}{2}\left[c_{\alpha+1,0}, c_{\alpha, 1}\right]$ & Gap closes at $\mu=v$, no phase transition & $\propto \mathbb{1}$ \\
& Degenerate at $\mu=v$ & $\left\langle a^{\dagger} a\right\rangle$ nonanalytic at $x_{c}$ \\
\hline$\mu a_{\alpha}, v a_{\alpha}^{\dagger}$ & Hamiltonian $H \neq 0:$ translationally invariant, critical at $x_{c}$ & $\propto \mathbb{1}$ \\
$\frac{i \mu}{2}\left[c_{\alpha, 0}, c_{\alpha, 1}\right], \frac{i v}{2}\left[c_{\alpha+1,0}, c_{\alpha, 1}\right]$ & Degenerate at $x_{c}$ & Nonanalytic at $x_{c}$ \\
\hline \hline
\end{tabular}

the form of a closing gap $(\Delta \rightarrow 0)$, a degeneracy of $\Delta$, or nonanalytic behavior of $\Delta$. These results are summarized in Table I.

With this paper, we suggest the possibility to detect certain system properties through an observation of the decoherent dynamics: Phase transitions in closed systems can be reflected in nonanalytic changes in the ADR $[25,26,28]$. More generally, since the ADR and other decay rates represent physical properties of the system, such nonanalyticities can be seen as the signature of a transition to a different dynamical regime. This suggests studying the phase diagram of steady- state correlation functions $\left\langle A(t) B\left(t^{\prime}\right)\right\rangle$, which reflect these dynamical transitions.

\section{ACKNOWLEDGMENTS}

The authors thank M. M. Wolf and T. Roscilde, B.H. thanks M. Lubasch, L. Mazza, M. C. Bañuls, N. Schuch, and A. Pflanzer for fruitful discussions. The authors would like to acknowledge financial support from the DFG within the Excellence Cluster Nanosystems Initiative Munich (NIM) and the EU project MALICIA under FET-Open Grant No. 265522.
[1] K. Southwell, V. Vedral, R. Blatt, D. Wineland, I. Bloch, H. J. Kimble, J. Clarke, F. K. Wilhelm, R. Hanson, and D. D. Awschalom, Nature (London) 453, 1003 (2008).

[2] P. W. Shor, Phys. Rev. A 52, 2493 (1995).

[3] F. Verstraete, M. M. Wolf, and J. Ignacio Cirac, Nat. Phys. 5, 633 (2009).

[4] B. Kraus, H. P. Büchler, S. Diehl, A. Kantian, A. Micheli, and P. Zoller, Phys. Rev. A 78, 042307 (2008).

[5] S. Diehl, A. Micheli, A. Kantian, B. Kraus, H. P. Buchler, and P. Zoller, Nat. Phys. 4, 878 (2008).

[6] S. Diehl, W. Yi, A. J. Daley, and P. Zoller, Phys. Rev. Lett. 105, 227001 (2010).

[7] M. Roncaglia, M. Rizzi, and J. I. Cirac, Phys. Rev. Lett. 104, 096803 (2010).

[8] W. Yi, S. Diehl, A. J. Daley, and P. Zoller, New J. Phys. 14, 055002 (2012).

[9] C. A. Muschik, E. S. Polzik, and J. I. Cirac, Phys. Rev. A 83, 052312 (2011).

[10] H. Krauter, C. A. Muschik, K. Jensen, W. Wasilewski, J. M. Petersen, J. I. Cirac, and E. S. Polzik, Phys. Rev. Lett. 107, 080503 (2011).

[11] F. Pastawski, L. Clemente, and J. I. Cirac, Phys. Rev. A 83, 012304 (2011).

[12] N. Syassen, D. M. Bauer, M. Lettner, T. Volz, D. Dietze, J. J. García-Ripoll, J. I. Cirac, G. Rempe, and S. Dürr, Science 320, 1329 (2008).

[13] S. Dürr, J. J. García-Ripoll, N. Syassen, D. M. Bauer, M. Lettner, J. I. Cirac, and G. Rempe, Phys. Rev. A 79, 023614 (2009).

[14] J.-J. García-Ripoll, S. Dürr, N. Syassen, D. M. Bauer, M. Lettner, G. Rempe, and J. I. Cirac, New J. Phys. 11, 013053 (2009).
[15] A. J. Daley, J. M. Taylor, S. Diehl, M. Baranov, and P. Zoller, Phys. Rev. Lett. 102, 040402 (2009).

[16] M. Kiffner and M. J. Hartmann, Phys. Rev. A 81, 021806(R) (2010); New J. Phys. 13, 053027 (2011).

[17] S. Diehl, A. Tomadin, A. Micheli, R. Fazio, and P. Zoller, Phys. Rev. Lett. 105, 015702 (2010).

[18] S. Sachdev, Quantum Phase Transitions (Cambridge University Press, Cambridge, UK, 1999).

[19] J. Eisert and T. Prosen, arXiv:1012.5013.

[20] E. M. Kessler, G. Giedke, A. Imamoğlu, S. F. Yelin, M. D. Lukin, and J. I. Cirac, Phys. Rev. A 86, 012116 (2012).

[21] T. Prosen, New J. Phys. 10, 043026 (2008).

[22] T. Prosen, J. Stat. Mech. (2010) P07020.

[23] T. Prosen and B. Žunkovič, New J. Phys. 12, 025016 (2010).

[24] S. R. Clark, J. Prior, M. J. Hartmann, D. Jaksch, and M. B. Plenio, New J. Phys. 12, 025005 (2010).

[25] M. Höning, M. Moos, and M. Fleischhauer, Phys. Rev. A 86, 013606 (2012).

[26] B. Horstmann, Ph.D. thesis, TU München, 2011.

[27] M. Žnidarič, J. Stat. Mech. (2010) L05002.

[28] M. Žnidarič, Phys. Rev. E 83, 011108 (2011).

[29] V. Eisler, J. Stat. Mech. (2011) P06007.

[30] X. Zhao, W. Shi, L.-A. Wu, and T. Yu, Phys. Rev. A 86, 032116 (2012).

[31] G. Lindblad, Commun. Math. Phys. 48, 119 (1976).

[32] H.-P. Breuer and F. Petruccione, The Theory of Open Quantum Systems (Oxford University Press, Oxford, 2007).

[33] M. A. Nielsen, Michael Nielsen's blog (2005).

[34] G. Wick, Phys. Rev. 80, 268 (1950). 
[35] C. V. Kraus, M. M. Wolf, J. I. Cirac, and G. Giedke, Phys. Rev. A 79, 012306 (2009).

[36] The exact steady-state CM for Eq. (54) differs from $\tilde{\Gamma}_{0}$ of Eq. (55) by $i 2 g^{2} \bigoplus_{n} \frac{\mu^{2}-v^{2}}{\left(k_{n}-l_{n}\right)^{2}+4\left|h_{n}\right|^{2}}\left[-\operatorname{Im}\left(h_{n}\right) \sigma^{z}+\frac{k_{n}-l_{n}}{2} \sigma^{x}\right]$, which vanishes as $g \rightarrow 0$.

[37] The ADR itself, $g^{2}\left(\mu^{2}+v^{2}\right)$, does not change at the phase transition. However, note that there actually is a large manifold of eigenvalues of the Liouvillian whose real part is $-g^{2}\left(\mu^{2}+v^{2}\right)$ but with different imaginary parts, taken from the set $\left\{\lambda_{ \pm}^{(n)}-\right.$ $\left.\lambda_{ \pm}^{(m)}\right\}$, where $\lambda_{ \pm}^{(n)}=\left(k_{n}+l_{n}\right) / 2 \pm \sqrt{\left(\frac{k_{n}-l_{n}}{2}\right)^{2}+\left|h_{n}\right|^{2}}$ and all four combinations of the subscripts \pm may occur. At the critical point, the discriminant vanishes, and each eigenvalue becomes (at least) fourfold degenerate.

[38] S. Braungardt, A. Sen(De), U. Sen, R. J. Glauber, and M. Lewenstein, Phys. Rev. A 78, 063613 (2008).

[39] D. Porras and J. I. Cirac, Phys. Rev. Lett. 92, 207901 (2004).

[40] A. Friedenauer, H. Schmitz, J. Glückert, D. Porras, and T. Schätz, Nat. Phys. 4, 757 (2008).
[41] H. Schmitz, A. Friedenauer, C. Schneider, R. Matjeschk, M. Enderlein, T. Huber, J. Glückert, D. Porras, and T. Schätz, Appl. Phys. B 95, 195 (2009).

[42] K. Kim, M.-S. Chang, S. Korenblit, R. Islam, E. E. Edwards, J. K. Freericks, G.-D. Lin, L.-M. Duan, and C. Monroe, Nature (London) 465, 590 (2010).

[43] J. W. Britton, B. C. Sawyer, A. C. Keith, C.-C. J. Wang, J. K. Freericks, H. Uys, M. J. Biercuk, and J. J. Bollinger, Nature (London) 484, 489 (2012).

[44] B. P. Lanyon, C. Hempel, D. Nigg, M. Müller, R. Gerritsma, F. Zähringer, P. Schindler, J. T. Barreiro, M. Rambach, G. Kirchmair, M. Hennrich, P. Zoller, R. Blatt, and C. F. Roos, Science 334, 57 (2011).

[45] J. T. Barreiro, M. Muller, P. Schindler, D. Nigg, T. Monz, M. Chwalla, M. Hennrich, C. F. Roos, P. Zoller, and R. Blatt, Nature (London) 470, 486 (2011).

[46] F. Marquardt and A. Püttmann, arXiv:0809.4403.

[47] D. J. Wineland, C. Monroe, W. M. Itano, D. Leibfried, B. E. King, and D. M. Meekhof, J. Res. Natl. Inst. Stand. Technol. 103, 259 (1998). 